\title{
El Dominio y su implicación para la Gestión de la Información *
}

\author{
Janet Tirador Ramos **
}

Artículo recibido:

16 de octubre de 2009.

Artículo aceptado:

11 de marzo de 2010.

\section{Resumen}

El siguiente trabajo presenta una aproximación teórica-conceptual que introduce los principales conceptos y teorías relacionadas con los dominios, con una visión basada en el Análisis de Dominios y las implicaciones que esto tiene para la Gestión de la Información. Se analiza tal relación a través de dos dimensiones fundamentales: las Comunidades y su similitud con los Sistemas, y la correlación que se puede dar entre los Once enfoques para analizar un dominio aportados por Hjørland (2002) y las etapas del Ciclo de Vida de la Información identificadas por Vizcaya (2003).

\footnotetext{
Investigación resultante del curso Gestión de Información del Doctorado en Documentación e Información Científica de la Universidad de Granada en conjunto con la AUIP.

** Universidad de Pinar del Río, Cuba. francis.sofia@fcsh.upr.edu.cu; francis.sofia@gmail.com

INVESTIGACIÓN BIBLIOTECOLÓGICA, Vol. 24, Núm. 50, enero/abril, 2010, México, ISSN: 0187-358X. pp. 49-60
} 
Palabras clave: Análisis de Dominio; Ciclo de Vida de la Información; Comunidades; Dominio; Gestión de Información; Sistemas.

\section{ABSTRACT}

The implication of domain for Information Management

Janet Tirador Ramos

A theoretical-conceptual focus is used to introduce the main concepts and theories related to domains, from a Domain Analysis perspective and its implications for Information Management. This relationship is analyzed through two fundamental dimensions: Communities and their similarity to Systems, and the correlation that can arise between the eleven approaches to analyze a domain provided by Hjørland (2002), and the stages of the Information Life Cycle identified by Vizcaya (2003).

Keywords: Domain analysis; Information Life Cycle; Communities: Dominion; Management Information; Systems.

\section{INTRODUCCIÓN}

$\mathrm{B}$ ajo un enfoque sociocognitivo aparecen nuevas propuestas teóricas y metodológicas como el Análisis de Dominio, tratado específicamente con base en la Ciencia de la Información. Dicho enfoque se centra en los significados de conceptos tales como: dominios, comunidad, discursos, que serán tratados más adelante, y desarrolla una visión que realza el valor de los contextos históricos y sociales en el espacio informacional.

Como paradigma gestado bajo la influencia de la Ciencia de la información es válido pensar que su corpus teórico general y la noción de dominio, entendida como comunidad unida por identidades comunes que poseen el sentido particular de representar una información, mantengan una correspondencia con el objetivo fundamental de esta ciencia, de participar y liderar todos los procesos y fases del ciclo de vida de la información.

Es un hecho que la visión de dominio parte de un enfoque social y contextual que encausa y delimita las necesidades, la búsqueda y la recuperación 
eficaz de información, de la comunidad que compone tal dominio. Estas acciones hacen que el dominio se convierta en un espacio útil para gestionar la información que poseen sus integrantes, a partir de una perspectiva holística y sistémica que module todos los flujos de información que fluyen entre ellos y entre las demás comunidades.

Pero la afirmación antes desarrollada no se encuentra explícitamente sustentada en la teoría del análisis de dominios, por lo que partiendo de esta problemática, es lógico que nos formulemos la siguiente interrogante ¿Cómo pueden integrarse teóricamente los Dominios y la Gestión de la información?

Para materializar esta interrogante partimos de una aproximación puramente exploratoria con el objetivo de describir la relación que existe entre los Dominios y la Gestión de la Información, y limitamos el alcance de la investigación a las siguientes dimensiones:

1. Las Comunidades y su similitud con los Sistemas, y

2. Los once enfoques para analizar un Dominio propuestos por Hjørland (2002), y su correlación con las etapas del Ciclo de Vida de la Información.

La descripción parte de una analogía entre estas dos áreas con el propósito de esclarecer los puntos lógicos que las unen, utilizando para ello el análisis documental y el análisis de información como técnicas de investigación, con la intención de que sirvan como inicio a nuevas líneas de investigación que cuenten con mayores niveles de profundidad.

\section{Apuntes SOBRE LOS DOMINIOS}

Hjørland es uno de los principales exponentes cuando de Análisis de Dominio se trata, pero aunque su teoría se ha enfocado hacia dicho análisis precisamente, un marco teórico conceptual referido exclusivamente a los dominios, no se encuentra desarrollado de forma clara en su literatura.

Para definir un Dominio, los autores (Hjørland; Albrechtsen, 1995) se refieren a una disciplina cientifica, un campo de estudios, o una comunidad discursiva agrupada por nexos comunes que se representan de forma dinámica.

Si partimos de que el análisis de los dominios encuentra sustento en la teoría de la actividad y del aprendizaje, se puede reconocer una equivalencia entre las comunidades discursivas y las comunidades de práctica identificadas por Wenger (1998) como un grupo social que tiene como objetivo común 
una interacción que le permite aprender y compartir el conocimiento que ambas comunidades poseen.

La diferencia que media entre esta analogía es que el dominio definido por Hjørland (Hjørland; Albrechtsen, 1995) ha evolucionado hacia el análisis de comunidades científicas, mientras que las comunidades de prácticas de Wenger (1998) mantienen un enfoque menos científico y más individual, al establecer que en estos espacios más informales también se intercambia y se aprende, aunque las acciones que realizan estas comunidades de práctica se pueden extender a otros tipos de grupos.

Por otra parte Swales (1990) profundiza en los elementos que definen a una Comunidad Discursiva y los identifica como un conjunto de personas con propósitos comunes, poniendo énfasis en las relaciones comunicativas que se establecen entre los miembros de un área determinada para intercambiar información y retroalimentarse a través de la interacción social del grupo.

En la teoría de Wenger (1998) también se evidencia explícitamente que el factor clave para intercambiar conocimientos y experiencias en una comunidad es la comunicación que se establece entre todos los integrantes del grupo. El autor propone además tres elementos que identifican a una comunidad de práctica y de aprendizaje, basado en su compromiso mutuo, la empresa conjunta y el repertorio compartido.

Se identifica como compromiso mutuo al establecimiento de reglas y deberes que permiten distinguir a los miembros de la comunidad como grupo social. Se señala también que en el momento en que se crean estas normas el grupo avanza hacia una empresa conjunta; es decir, se crea el medio para que los miembros se interrelacionen y definan las concepciones comunes y los objetivos que los unen.

Luego que la comunidad interactúe, establezca sus normas y llegue a un consenso de cómo identificar y tratar su objetivo fundamental, se habla de un tercer elemento haciendo referencia a los repertorios compartidos, que no son más que la totalidad de los recursos producidos y utilizados por el grupo que marcan su visión propia del espacio de conocimiento en el que se desempeñan.

Siguiendo esta misma línea conceptual, para identificar una comunidad determinada, Hjørland y Hartel (2003) se refieren a tres dimensiones fundamentales que constituyen básicamente la armadura teórica y conceptual de un dominio, definido éste por una teoría ontológica y conceptual de los objetos de la actividad humana, una teoría y conceptos epistemológicos del conocimiento y las formas de lograr su obtención. Por último, hacen también referencia a los conceptos sociológicos de las personas que conforman el grupo.

En la dimensión ontológica se analizan las propiedades, estructuras y procesos de objetos de la actividad humana y su relación en cualquier área de 
la realidad (Smith, 2004). Es en este punto en el que se analiza y se trata de explicar la realidad de un dominio y su estructura, para establecer los conceptos relevantes del dominio y sus relaciones. Este conjunto de conceptos es denominado como Universo del Discurso (Gruber, 2001), el cual facilita la comunicación entre los integrantes del dominio. Este principio es una forma mediante la cual se puede organizar de forma intelectual el conocimiento y la información que posee la comunidad al ofrecer explicaciones de términos como: áreas, dominios, elementos, campos, tipos, objetos, problemas, asuntos temáticos, materias.

Estas teorías son totalmente dinámicas en tanto que se enriquecen o cambian según lo hagan también las estructuras sociales y conceptuales marcadas por las transformaciones y las visiones de un objeto a través de las especialidades en temas pluri, multi, inter y transdisciplinares.

Mientras que en una segunda dimensión se analizan los aspectos epistemológicos que identifican el dominio, con el objetivo de estudiar la información y el conocimiento que poseen los integrantes del grupo, su lenguaje, tradiciones, análisis teóricos del campo de conocimiento, los paradigmas bajo los cuales enfocan su dominio, y las distintas visiones, métodos o valores que poseen, utilizando para ello como una de las herramientas el análisis de su producción de la literatura científica y la posterior representación en ontologías (en el sentido terminológico) a través de taxonomías, con el objetivo de identificar la cultura y las prácticas de un dominio.

Los autores Hjørland y Hartel (2003) presentan una serie de ejemplos de teorías epistemológicas que pueden influir en un dominio, algunas de las cuales son: constructivismo, racionalismo crítico, empiricismo, epistemología feminista, hermenéutica, fenomenología, pragmatismo, racionalismo, etc. Para identificar estos aspectos epistemológicos en los dominios, los autores listan algunas palabras clave tales como: metateorias, movimientos, paradigmas, $f$ losofías de la disciplina en cuestión, escuelas, sistemas de pensamiento o investigación, tradición académica y puntos de vista (cognitivo) entre otras. Por lo que para delimitar el dominio se parte de un componente histórico que enriquece las observaciones empíricas y permite separar los fenómenos de la realidad.

En el caso de la dimensión referida a los aspectos sociológicos se identifican una serie de elementos del grupo analizado, como integrantes sociales de una realidad que se relaciona con los objetos; estos son: la disciplina y subdisciplina que imparte o investiga, las comunidades del discurso, la profesión y la especialidad, así como aspectos más específicos de la vida cotidiana tales como los hobbies del grupo, etcétera.

La interrelación entre la dimensión ontológica, la dimensión epistemológica y la social es vital para poder llevar a cabo un correcto estudio del dominio 
seleccionado para la investigación. A su vez cada comunidad es analizada de forma diferente y desarrolla estas tres dimensiones atendiendo a criterios disímiles, ya que sus discursos y sus metas no son iguales, así como también varía su constitución, su influencia paradigmática y el conocimiento que posee cada integrante y la forma de explicitarlo, por lo que se puede afirmar que la información que genera y los medios para gestionarla también son diferentes, ya que dicho proceso debe respetar cada particularidad de los dominios.

Para llevar a cabo el análisis de una comunidad discursiva determinada Hjørland (2002) define once enfoques o métodos, basados en la construcción de:

1. Guias de fuentes de información sobre el dominio, que representen la producción científica de un dominio, en particular mediante taxonomías que marquen las fuentes más significativas.

2. Clasificaciones especializadas que contengan los conceptos fundamentales del dominio y sus relaciones semánticas.

3. Indización y recuperación de la información representada en documentos especializados.

4. Estudios empíricos de los usuarios, sus necesidades y las comunidades a las que pertenecen.

5. Estudios bibliométricos para visualizar los mapas de la ciencia y las relaciones pormenorizadas entre los documentos.

6. Estudios históricos del dominio.

7. Estudios sobre la estructura de los documentos y la arquitectura de la información que representan la comunicación, apoyados en la dimensión histórica y social.

8. Estudios epistemológicos y críticos sobre la disciplina o temática que representa el dominio y que pautan la selección, organización y recuperación de la información.

9. Estudios terminológicos, semántica de las bases de datos y estudios del discurso.

10. Estudios sobre la estructura y las instituciones en cuanto a comunicación científica.

11. Cognición científica, conocimiento de expertos en Inteligencia Artificial que aporten los esquemas mentales de un dominio.

Cuando se analiza un dominio atendiendo las tres dimensiones identificadas y los once enfoques que las materializan, se pueden obtener resultados que repercutan en la elaboración, a través de técnicas bibliométricas, de mapeos de la ciencia, y en la organización del conocimiento y la Gestión de Información en dichas comunidades. 


\section{APUNTES SOBRE LA GESTIÓN DE LA INFORMACIÓN}

Si se analiza el alcance del concepto de información aportado por diversos autores desde sus perspectivas paradigmáticas se hace evidente que ésta posee dos componentes fundamentales, uno objetivo, donde se representan las estructuras significantes, y uno componente, que depende completamente del sujeto que analiza dichas estructuras y les aporta un significado, evidenciando el rol, imprescindible dentro del concepto, de un receptor que dota de significados a los datos que modifican su conocimiento y conducta en un contexto determinado.

En la actualidad la información se ha convertido en un factor clave para el desarrollo no sólo de los individuos sino de todo tipo de comunidad, por lo que su gestión se hace necesaria en cualquier tipo de institución con el objetivo de conocer la información que se posee y de darle seguimiento, con los recursos necesarios, a los flujos que marcan el ciclo de vida de la información. Estas actividades revelan los dos principios esenciales que sostienen la Gestión de la Información: la Teoría de Sistemas y la Teoría del ciclo de vida (Ponjuán, 2007).

Se entiende por sistema un conjunto de elementos interrelacionados y regidos por normas propias, de modo tal que pueden ser vistos y analizados como una totalidad (Barite, 2000). Unido a este concepto se presenta la visión de que los sistemas están dotados de una dinámica que les permite interactuar con sus componentes internos y a su vez actuar sobre el medio que lo rodea, con la función de adaptarse, aprender, auto-organizarse, retroalimentarse y así poder cumplir los objetivos propuestos.

La Teoría de los Sistemas le aportó a la Gestión de la Información la estructura y la interacción que se establece entre los elementos que coexisten bajo una agrupación que realiza acciones enfocadas hacia resultados determinados; es decir, define el espacio y los componentes que utilizarán las fases del ciclo de vida de la información para su desarrollo.

Por su parte el Ciclo de Vida de la Información revela los procesos por los que transita la información y que, como modelo, puede ser adaptado a cualquier tipo de sistema. Existen diversas representaciones sobre las etapas del Ciclo de Vida de la Información, una de las más completas de las cuales es la aportada por Vizcaya (2003), quien esquematiza de forma holística y sistémica todas las actividades y flujos que ocurren, representadas en la siguiente figura: 


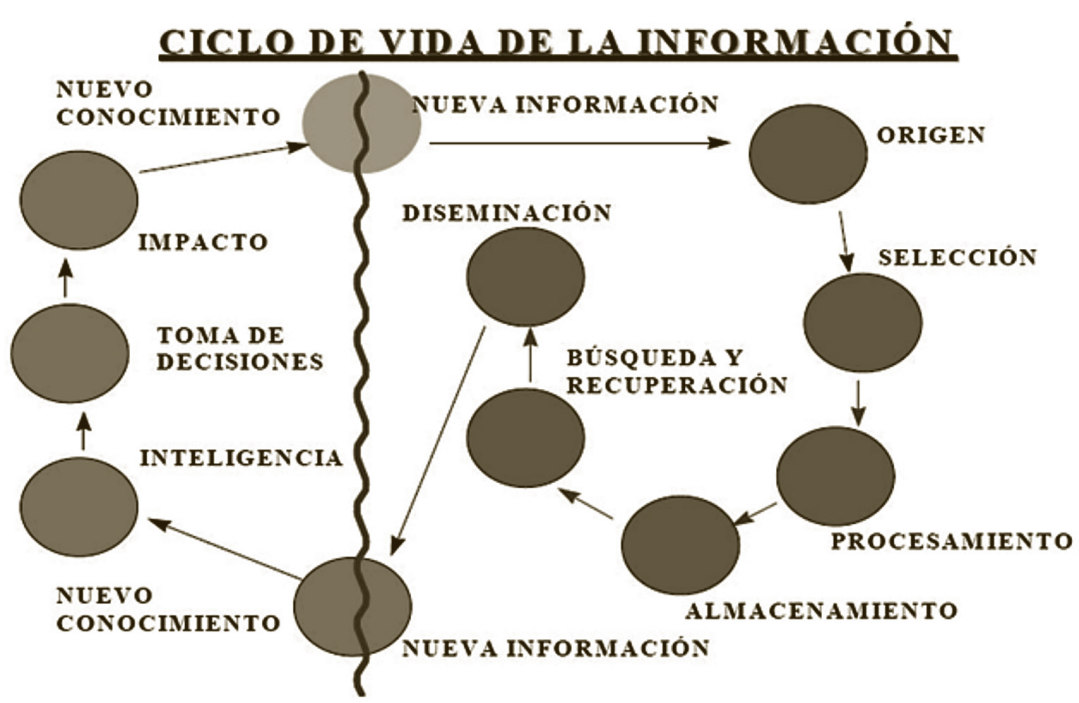

Fig.1. Ciclo de Vida de la Información. (Vizcaya, 2003)

Esta representación enfatiza las etapas relacionadas con el procesamiento de la información, que se imponen como núcleo de las posteriores facetas que identifican la repercusión social que obtendrá dicho procesamiento.

\section{EL DOMINIO Y LA GESTIÓN DE LA INFORMACIÓN}

Al realizar un análisis del concepto aportado por Swales (1990) sobre una Comunidad Discursiva, se demuestra que concuerdan muchos de los elementos que destaca conceptualmente cuando define un sistema. El autor destaca que las personas que integran una comunidad poseen identidades comunes, mantienen intereses y creencias similares o simplemente comparten una misma cultura, región geográfica y lenguaje, que les facilita una interrelación comunicativa y les permite intercambiar información. Características muy similares que se manejan en la Teoría de Sistemas al referirse a un sistema como un grupo de elementos que interaccionan e intercambian información entre ellos y su ambiente, y se enfocan hacia resultados específicos.

Esta posible similitud conceptual nos permite tratar una Comunidad Discursiva como un sistema, teniendo presente el Principio de la Relatividad y la composición o las funciones del dominio que definirán el nivel de subordinación entre los sistemas y subsistemas que se puedan presentar. Esto permite 
además suponer que todo dominio posee las propiedades de homeostasis, entropía, permeabilidad, centralización y descentralización, estabilidad y adaptabilidad, que le son inherentes a los sistemas.

Es válido añadir que en las comunidades se pueden identificar, al igual que en la teoría de sistemas, tres características estructurales básicas: los elementos que los componen, las relaciones entre los mismos y los límites que determinan los elementos que pertenecen o no al sistema (Ponjuán; Mena; Villardefrancos; et. al, 2004). Estas particularidades pueden utilizarse, al igual que las propiedades de los sistemas, identificados anteriormente, para caracterizar cualquier tipo de dominio.

La relación que se puede evidenciar entre comunidad y sistema, no sólo está dada en la analogía de cada una de sus funciones, sino también en la utilización del enfoque sistémico como herramienta para modelar y describir el análisis estructural aplicable a cualquier grupo, bajo la visión de Boulding (1993) de que cualquier cosa no es un caos, es un sistema. Toda comunidad puede ser observada holísticamente a través de sus interacciones y los efectos que puede producir en el dominio.

Pues el estudio de un dominio permite definir, analizar y representar las características que determinan una interrelación entre sus integrantes. Permite analizar el tipo de comunicación que se establece o el grado de importancia que le puede aportar una comunidad a un tema; es decir, se puede esbozar cómo los integrantes entienden un área determinada y qué significado le aportan a diversos datos o mensajes, valiéndose para su representación de ontologías, utilizadas desde el punto de vista de la Inteligencia Artificial, o simplemente permite, a través de aspectos sociales, establecer la relación o retroalimentación que se establece entre sus miembros, lo cual sería muy útil para definir los flujos de información en una institución.

Una de las mejores formas para lograr una adecuada Gestión de la Información que fluye en un dominio determinado es analizar y estudiar las particularidades y estructuras de dichas comunidades, las que al mantener una comunicación entre sus miembros y una relación basada en el aprendizaje, propician el espacio que el propio dominio necesita para identificar, compartir almacenar y codificar información y conocimientos. Para lo cual se apoyarían como elemento clave, en los repertorios compartidos, que poseen el corpus documental que identifica toda la información y el conocimiento que fluye en el dominio.

Si se analiza la teoría del aprendizaje situado (Lave; Wenger, 1991), aplicada a las comunidades de práctica, se vuelve un hecho que el factor clave del aprendizaje está vinculado con la colaboración y el intercambio de información, traducida no sólo en datos con estructura y significado, sino también 
en experiencias y habilidades de forma participativa. De manera tal que no sólo se pueden aplicar en esta teoría las distintas fases del ciclo de vida de la información, sino que también le puede aportar a la teoría de la Gestión de la Información la dinámica del aprendizaje participativo bajo el enfoque social de la información.

Por otra parte, al realizar un análisis comparativo entre los once enfoques propuestos por Hjrland (2002) y las fases del ciclo de vida de la información, se pueden establecer algunas similitudes, ya que los tres primeros métodos coinciden con facetas específicas del ciclo. Al aplicar los tres primeros enfoques, se obtiene como resultado, primero una guía que contiene todas las fuentes de información que existen sobre el dominio, y segundo una taxonomía con los conceptos fundamentales de dichas fuentes, lo que no sólo representa una serie de palabras clave que identifican a un grupo, sino una clasificación de forma jerárquica del entendimiento de un dominio, para concluir con la indización y la recuperación de la información que se representó anteriormente. Estas actividades evidencian de forma explícita que existe una concordancia entre los métodos explicados y los procesos de selección, clasificación, indización y recuperación que ocurren en el Ciclo de Vida de la Información.

Estos tres primeros enfoques posibilitarán armar un corpus informacional para poder realizar los ocho restantes, que se refieren a estudios de usuarios, análisis de la estructura de los documentos y la terminología utilizada, la identificación de los enfoques epistemológicos bajo los cuales se desarrollan, y también permitirá aplicar técnicas bibliométricas para mapear ese dominio.

Es valido aclarar que si cada uno de los estudios, aunque no coincidan explícitamente con etapas formalizadas del Ciclo de Vida de la Información, se realizan en la mayoría de las etapas, se hará necesario analizar, por ejemplo, en la fase de Selección, los usuarios y sus necesidades, para poder adquirir las fuentes de información.

La etapa de Procesamiento no sólo atiende los resultados de los estudios de usuarios para poder realizar sistemas de representación y organización que le sean pertinentes para luego recuperar la información, sino que se apoya en fuertes estudios terminológicos, históricos y sociales de manera tal que éstos reflejen el significado de la información sin perder el componente contextual.

Los estudios bibliométricos suelen realizarse, al igual que los demás, en cualquiera de las etapas del Ciclo, con varios propósitos, uno de los cuales es analizar y mapear las relaciones que puedan existir entre autores que representan una temática especifica dentro de un dominio determinado, a través 
de análisis de cocitación, unidos a las técnicas de escalamiento multidimensional (MDS).

Si los resultados de cada uno de los estudios propuestos por Hjørland (2002) fueran socializados o diseminados generarían una información que de seguro podría ser aplicada, por sus efectos analíticos y puntuales, para la toma de decisiones que tendrían una repercusión social, ya sea en la comunidad analizada o en otros dominios relacionados, lo que podría dar inicio, nuevamente, a todo un ciclo que tratara la nueva información.

La tendencia del análisis presentado indica que los resultados de los once enfoques complementan en su mayoría todas las fases del ciclo de vida de la información, de manera que cada uno puede ser utilizado como una herramienta que facilita y apoya la Gestión de la Información.

\section{Conclusiones}

1. Cada dominio representa una Comunidad con un conocimiento y un discurso propio y dinámico, por lo que debe analizarse de forma particular.

2. El análisis de un dominio se enfoca en una dimensión social, que se materializa en los once métodos aportados para su estudio.

3. Las propias actividades de la Gestión de la Información revelan los dos principios esenciales que la sostienen: la Teoría de Sistemas y la Teoría del Ciclo de Vida de la Información, respetando y representando las peculiaridades de cada dominio.

4. Al realizar un análisis del concepto de Comunidad Discursiva se demuestra que muchos de los elementos que destaca conceptualmente concuerdan con la definición de un sistema.

5. La tendencia del análisis presentado indica que los resultados de los once enfoques complementan en su mayoría todas las fases del Ciclo de Vida de la Información, de manera que pueden ser utilizados como una herramienta que facilita y apoya la Gestión de la Información.

\section{BiBLIOGRAFÍA}

(Barite, 2000) Barite, M. (2000), KO Dictionary (en línea), (consultado: ene, 2010), disponible en: http://www.eubca.edu.uy/diccionario/letra_s.htm

(Boulding, 1993) Boulding, K. (1993), Chaos \& complexity, $\mathrm{VCH}$, Weinheim. 
(Gruber, 2001) Gruber, T. R. (2001), What is an Ontology, (en línea), (consultado: dic, 2006), disponible en: http://www-ksl.stanford. edu/kst/what-is-an-ontology.html

(Hjørland; Albrechtsen, 1995) Hjørland, B; Albrechtsen, H (1995), "Toward a new horizon in Information Science: Domain Analysis", en Journal of the American Society for Information Science, 45(6): 400-425.

(Hjørland, 2002) Hjørland, B (2002), "Domain Analysis in Information Science: Eleven Approaches - Traditional as Well as Innovative", en Journal of Documentation, 58(4): 422-462.

(Hjørland; Hartel, 2003) Hjørland, B; Hartel, J (2003), "Afteword: Ontological, Epistemological and Sociological dimensions of domains", en Knowledge Organization, 30 (3): 239-245.

(Lave; Wenger, 1991) Lave.J; Wenger E. (1991), Situated Learning. Legitimate peripheral participation, Cambridge, Cambridge University Press.

(Ponjuán, 2007) Ponjuán Dante, G (2007), Gestión de información: Dimensiones e implementación para el éxito organizacional, España: Ediciones Trea, 157 p.

(Ponjuán; Mena; Villardefrancos; et. al, 2004) Ponjuán Dante, G; Mena, M; Villardefrancos, M.C; et. al (2004), Sistemas de información: Principios y aplicaciones, La Habana, $128 \mathrm{p}$.

(Smith, 2004) Smith, B. (2004), Ontology, en Florido, L. (ed), The Blackwell guide to the philosophy of computing and information, Oxford, Blackwell publishing, 155-166.

(Swales, 1990) Swales, J. (1990), Genre analysis: English in academic and research settings, Cambridge, UK, Cambridge University Press.

(Vizcaya, 2003), Vizcaya Alonso, D (2003), Información: Procesamiento de contenido, La Habana, Editorial Félix Varela, 187 p.

(Wenger, 1998) Wenger, E. (1998), Communities of Practice: Learning, Meaning, and Identity, Cambridge University Press. 